\title{
EEG correlates of working memory performance in females
}

Yuri G. Pavlov ${ }^{1,2^{*}}$ and Boris Kotchoubey ${ }^{1}$

\begin{abstract}
Background: The study investigates oscillatory brain activity during working memory (WM) tasks. The tasks employed varied in two dimensions. First, they differed in complexity from average to highly demanding. Second, we used two types of tasks, which required either only retention of stimulus set or retention and manipulation of the content. We expected to reveal EEG correlates of temporary storage and central executive components of WM and to assess their contribution to individual differences.

Results: Generally, as compared with the retention condition, manipulation of stimuli in WM was associated with distributed suppression of alpha1 activity and with the increase of the midline theta activity. Load and task dependent decrement of beta1 power was found during task performance. Beta2 power increased with the increasing WM load and did not significantly depend on the type of the task. At the level of individual differences, we found that the high performance (HP) group was characterized by higher alpha rhythm power. The HP group demonstrated task-related increment of theta power in the left anterior area and a gradual increase of theta power at midline area. In contrast, the low performance (LP) group exhibited a drop of theta power in the most challenging condition. HP group was also characterized by stronger desynchronization of beta1 rhythm over the left posterior area in the manipulation condition. In this condition, beta2 power increased in the HP group over anterior areas, but in the LP group over posterior areas.

Conclusions: WM performance is accompanied by changes in EEG in a broad frequency range from theta to higher beta bands. The most pronounced differences in oscillatory activity between individuals with high and low WM performance can be observed in the most challenging WM task.
\end{abstract}

\section{Background}

The ability to retain information in memory for a short period of time is critical for numerous cognitive tasks including planning, verbal competence, spatial orientation, mental manipulations of objects and many others [1-3].

According to Baddeley and Hitch's [4] model, the structure of working memory (WM) consists of several components. One of them is responsible for temporary storage of information in modality-specific buffers. Another key component, the central executive, is considered to be a set of tools designed to maintain the active

\footnotetext{
*Correspondence: pavlovug@gmail.com

${ }^{1}$ Institute of Medical Psychology and Behavioural Neurobiology,

University of Tübingen, Tübingen, Germany

Full list of author information is available at the end of the article
}

representation of memory trace, to control attention and to preserve the latter from interference caused by irrelevant stimuli $[5,6]$.

A number of neuroimaging studies demonstrated that maintenance of information in WM engages a broad network of neural structures mostly including prefrontal cortex, parietal and temporal areas [2, 7]. Whereas storage buffers represent information received from sensory inputs in posterior regions, the prefrontal cortex sustains and transforms this information and organizes executive processes of working memory [8]. Existing research highlights the importance of the fronto-parietal network activation in working memory processes, especially in high demanding tasks [9-13]. Apparently, individual differences in working memory capacity are also determined by fronto-parietal white matter connectivity [14]. 
Features of the processes presumed by Baddeley and Hitch's model of WM cannot be characterized only by spatial distribution of brain activation. Qualitatively different information about these processes can be obtained from studies of neuronal oscillatory activity as an energyefficient mechanism for temporal coordination of cognitive processes [15].

An increase of frontal midline theta rhythm (FMT) frequently accompanies such processes as nonspecific attention and WM [16-19]. The results of earlier studies often define FMT as the most plausible phenomenon reflecting an activation of central executive components of WM [20]. Several attempts to isolate central executive components from temporary storage components by including tasks requiring mental manipulations support hypothesis of the link between FMT and the executive control [21-23]. Several studies demonstrated the activation of fronto-parietal executive control system during retention in WM [24-26]. Moreover, some authors report increasing fronto-parietal synchronization with stronger engagement of central executive components [19]. Induced coupling of theta rhythm between frontal and parietal cortical regions by transcranial alternating current stimulation (tACS) resulted in improved visual WM performance, while the induced decoupling lead to WM deterioration [27].

Changes in alpha activity also show parametrical increase related to working memory load [28-30]. Increasing power of alpha rhythm is frequently interpreted as a mechanism for filtration and for suppression of the cortical areas irrelevant to the current task [30-32].

The role of beta activity in working memory processes is still not sufficiently investigated. Thus the activity particularly in the low beta band $(\sim 13-20 \mathrm{~Hz})$ was found to increase during retention in WM [18, 33-35]. A parametrical increase of low beta with the increasing of memory set size was also observed [18, 33]. A comparison of retention condition with the conditions where participants were instructed to manipulate objects in WM showed that gradually increasing task complexity was related with a decrease of low beta activity [22].

Data of several studies suggest that the main contribution to individual differences in WM is made by the ability to control attention or executive control [36-38]. However, despite extensive research of WM in the recent 20 years, there is no clarity as regards the electrophysiological correlates mechanisms of individual differences in WM performance. The existing research (both general and differential psychological) have some limitations that restrict the possibility to explain the actual relationship between brain activity and WM performance.
First of all, most WM studies have used the n-back paradigm [39-41]. This kind of task engages multiple WM processes including retention of the stimuli set presented at the previous step, comparison between the first item of the memorized set and the new one, making decision about correctness of the comparison, and updating the content of WM. In this paradigm, it is difficult to clearly separate retention from the central executive components of WM.

Second, the level of difficulty of the task is usually moderate and thus does not present a big challenge for people with average WM abilities. There are studies dedicated to the investigation of EEG in WM tasks with several levels of difficulty $[18,40,42,43]$. In the studies mentioned above the number of steps did not exceed three (3-back) $[40,41]$. Some researchers applied other paradigms with gradually increasing difficulty of tasks for assessing WM performance $[17,18,23]$. But these paradigms either did not include any manipulation task $[17,18]$, or their difficulty level was rather low [23].

Finally, the existing studies aimed to discover electrophysiological correlates of individual differences in WM were based on a sample size not exceeding 14 participants in each group [18, 40,44]. An analysis of typical effect sizes indicates that at least twice larger groups would be necessary to reliably evaluate the differences between high- and low-performers.

In this paper we used highly demanding tasks which should give us the opportunity to distinguish EEG activity of individuals with different levels of WM performance. Additionally, using two types of tasks, which required either only retention of stimulus set or manipulation of content, we expected to reveal EEG correlates of temporary storage and central executive components of WM and to assess their contribution to individual differences.

The hypotheses of the study were as follows:

1. Motivated by the previous studies we expected significant relationships between WM performance and oscillatory activity in theta and alpha frequency bands;

2. Particularly, we supposed that frontal theta rhythm power is strongly related to the WM load;

3. We expected that storage components of working memory play less important role in individual differences than executive components. Specifically, we assumed that no individual differences would be found in the simple retention conditions;

4. Additionally, we hypothesized that the most challenging condition would best separate between low and high performers; 


\section{Methods}

\section{Participants}

Due to a strong gender disproportion in the initial sample, only data of female participants were included into the present study. All participants were Russian native speakers. Furthermore, a subsequent analysis revealed five EEG records with an excessive amount of artefacts (i.e., $<20$ artifact-free epochs in at least one condition). Thus, 65 female participants (mean age $=20.92$, $\mathrm{SD}=2.96)$ were included to the final sample. The participants had normal or corrected-to-normal vision and no history of neurological or mental diseases.

\section{Stimuli}

Sets of Russian alphabet letters written in capital were used as stimuli. The letters had been selected randomly and had random order and no repetitions in the sets. Each trial consisted of seven consecutive events. An analogue using Latin letters and English words is shown in Fig. 1.

A trial always began with an exclamation mark presented for $200 \mathrm{~ms}$, which was followed by a fixation cross for $3000 \mathrm{~ms}$. Participants were instructed to fixate the cross when it appeared in the center of the screen. At the next step the word "forward" or "alphabetical", presented for $500 \mathrm{~ms}$, instructed participants whether they would have to memorize the original set as it was presented (retention task) or to memorize it after mental recombination of the letters in the alphabetical order (manipulation task). After that, sets of 5,6 or 7 letters were demonstrated for $3000 \mathrm{~ms}$ followed by a delay period where a fixation cross was demonstrated for $6500 \mathrm{~ms}$. At the end of this delay period, a randomly chosen letter from the previously presented set appeared on the screen together with a digit that represented the serial number of this letter. The letter-digit combination was presented for $1000 \mathrm{~ms}$. Participants were asked to press a specified button of a computer mouse if the presented letter had the corresponding serial number either in the original set (in the retention task), or in the set merging as a result of alphabetic recombination (in the manipulation task). The other mouse button had to be pressed if the serial number of the presented letter was incorrect. The two buttons were attributed to correct and wrong probes in a counterbalanced order. The probe was correct in $50 \%$ of the trials, and the order of correct and incorrect probes was random. The next trial started after an interval that varied between 5000 and $5500 \mathrm{~ms}$.

Thus, the experiment entailed six different conditions: memorizing 5, 6 or 7 letters in the alphabetical or forward order. Each condition had 20 consecutive trials. These six blocks with 20 trials were presented in a random order. A short practice block of 6 trials was given immediately before the main experiment.

During the experiment, the participants were seated in a comfortable armchair in front of a computer screen in a dark room. Stimuli were presented in white color on a black background in the center of the screen by using PsyTask software (Mitsar Ltd.). The distance to the screen was $1 \mathrm{~m}$ and the size of the letters was $1.2^{\circ} \times 1.2^{\circ}$.

All participants were subdivided into two groups separated by the median of their mean performance across all tasks. The groups are referred to as high performance (HP; $\mathrm{N}=32$ ) and low performance (LP; $\mathrm{N}=33$ ) groups. The percentage of correct answers was used for behavioral data analysis. A repeated measures ANOVA with the between-subject factor Group (HP, LP) and the withinsubject factors Task (retention, manipulation) and Load $(5,6$, or 7 letters $)$ was applied.

\section{EEG recording and analysis}

The EEG was recorded from 19 electrodes arranged according to the 10-20 system using Mitsar-EEG-201 amplifier and referred to the average earlobe. Two additional electrodes were used for horizontal and vertical EOG. EEG data were acquired with $500 \mathrm{~Hz}$ sampling

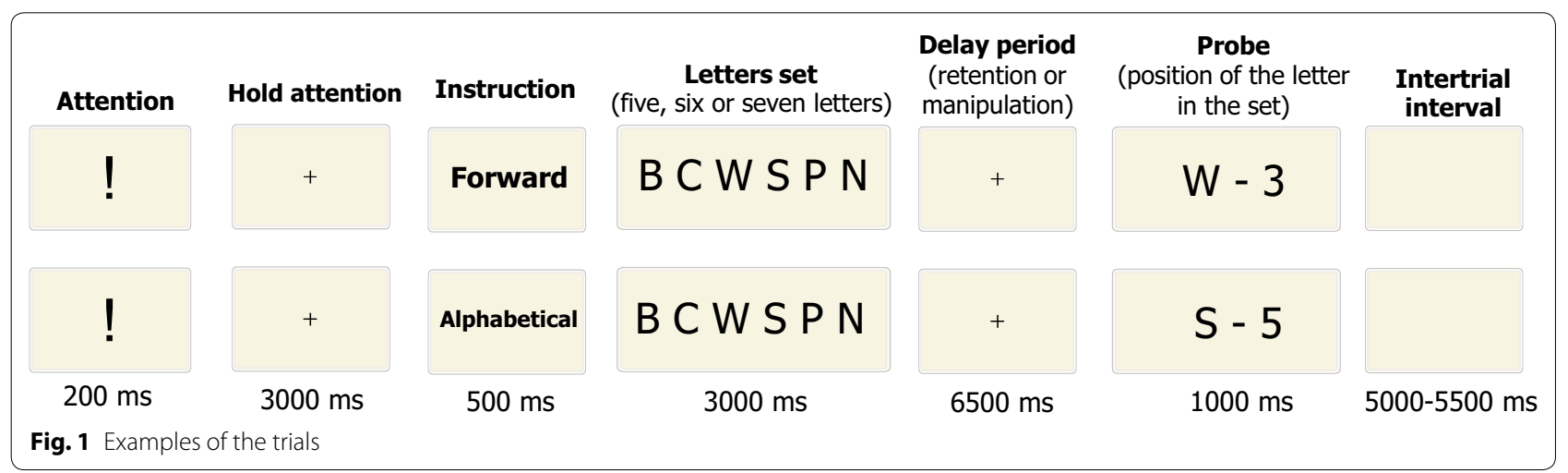


frequency, $0.16 \mathrm{~Hz}$ high pass filter and $70 \mathrm{~Hz}$ low pass filter.

Frequency bands for EEG analysis were defined using individual alpha frequency (IAF) as follows: theta $=($ IAF- $6 \mathrm{~Hz}$ to IAF- $2.5 \mathrm{~Hz})$, alpha1 $=($ IAF$2.5 \mathrm{~Hz}$ to IAF), alpha2 = (IAF to IAF $+2.5 \mathrm{~Hz})$, beta $1=(\mathrm{IAF}+2.5-20 \mathrm{~Hz})$, beta $2=(20-30 \mathrm{~Hz})$. The IAF was determined on a 3 min EEG recorded at rest with eyes closed.

Segments of raw EEG recorded during the interval from 500 to $6500 \mathrm{~ms}$ of the delay period were analyzed. These segments were filtered between 0.5 and $30 \mathrm{~Hz}$, and a $50-\mathrm{Hz}$ notch filter was applied. The segments were subdivided into 2-s epochs. A fast Fourier transformation (FFT) was performed in each epoch. Ocular artefacts were corrected by using independent component analysis (ICA) followed by visual EEG inspection for remaining artefacts (see Table 1 for number of artifact-free epochs per EEG condition). These operations were performed in EEGlab toolbox. Spectral power densities for each frequency bands were calculated using Fieldtrip toolbox.

Spectral power data were statistically analyzed by using two independent mixed-design ANOVAs. The first analysis involved mean power values in four regions of interest (ROI): left (Fp1, F7, F3) and right (Fp2, F8, F4) anterior areas, left $(\mathrm{T} 5, \mathrm{P} 3, \mathrm{O} 1)$ and right $(\mathrm{T} 6, \mathrm{P} 4, \mathrm{O} 2)$ posterior areas. This analysis included a between-subject factor Group (HP, LP) and the within-subject factors Task (retention, manipulation), Load (5 vs. 7 letters), Hemisphere (left, right) and Site (anterior, posterior).

The second ANOVA of mean power values at the midline $(\mathrm{Fz}, \mathrm{Cz}, \mathrm{Pz})$ was used exclusively for planned testing the hypothesis about the dynamics of the theta rhythm. The results in the other frequency bands were not analyzed. The ANOVA included factors Group (betweensubject), Task and Load (within-subject). All statistical calculations were performed by using SPSS package.

\section{Results}

\section{Behavioral results}

Participants performed with a general mean accuracy of $78.5 \pm 0.9 \%$. Mean accuracies for each condition are shown in Fig. 2.

The main effects of Task $[\mathrm{F}(1,63)=108.1, \mathrm{p}<0.0001$, $\left.\eta^{2}=.632\right]$ and Load $[\mathrm{F}(2,126)=49.69, \mathrm{p}<0.0001$,

Table 1 Number of artifact-free epochs per EEG condition

\begin{tabular}{lll}
\hline & Retention & Manipulation \\
\hline 5 letters & $52.4 \pm 8.02(21)$ & $53.4 \pm 6.63(27)$ \\
7 letters & $54.2 \pm 6.41(25)$ & $53.5 \pm 6.87(31)$ \\
\hline
\end{tabular}

Mean \pm standard deviation (minimum)

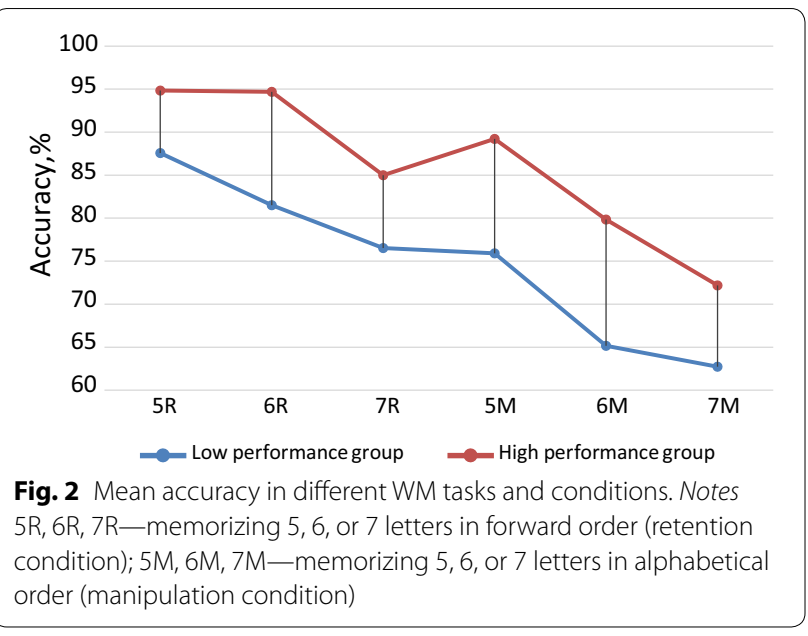

$\left.\eta^{2}=.441\right]$ as well as their interaction of the factors $\left[F(2,126)=5.606, p=0.005, \eta^{2}=.082\right]$ were obtained. A pairwise comparison between load levels separately for alphabetical and forward conditions showed highly significant differences $(\mathrm{p}<0.0001)$ for all pairs but two. First, there was no difference between the performance in 5and 6-letter conditions in the forward order $(\mathrm{p}=0.191)$. Second, the differences were less pronounced in the comparison between 6 and 7 letters in the alphabetical order $(\mathrm{p}=0.011$; not significant after Bonferroni correction). For this reason, and in order to avoid potential problem with sphericity in statistical measures, the 6-letters condition was excluded from the EEG analysis.

The mean performance accuracy in the high and low performance groups was $84.9 \pm 0.5$ and $71.9 \pm 1.1 \%$, for HP and LP, respectively $[\mathrm{F}(1,63)=87.26, \mathrm{p}<0.0001$, $\left.\eta^{2}=.581\right]$. The difference between HP and LP did not substantially differ as a function of Task and Load.

\section{Electrophysiological results \\ Theta}

The theta rhythm had lower power in anterior areas in comparison with posterior areas (main effect of Site, see Table 2). Also, the power was higher over the left than the right hemisphere (main effect of Hemisphere). Furthermore, the theta power decreased with the increasing WM load at all ROIs except the right anterior one (Load $\times$ Site $\times$ Hemisphere interaction).

Across the whole sample, the theta power tended to be higher in the manipulation task than in the retention task. As depicted in Fig. 3, this effect was more pronounced at anterior than posterior areas (Task $\times$ Site interaction) and also more pronounced over the left than the right hemisphere (Task $\times$ Hemisphere interaction). The analysis also revealed a four-way interaction between Task, Site, Hemisphere and Group. Specifically, in the HP 
Table 2 Results of the ANOVA with the factors Task $\times$ Load $\times$ Hemisphere $\times$ Site $\times$ Group

\begin{tabular}{|c|c|c|c|c|c|c|c|c|c|c|c|c|c|c|c|}
\hline & \multicolumn{3}{|l|}{ Theta } & \multicolumn{3}{|c|}{ Alpha1 } & \multicolumn{3}{|c|}{ Alpha2 } & \multicolumn{3}{|l|}{ Beta1 } & \multicolumn{3}{|l|}{ Beta2 } \\
\hline & $\mathbf{F}$ & $\mathrm{p}$ & $\eta^{2}$ & $\mathrm{~F}$ & $p$ & $\eta^{2}$ & $\mathrm{~F}$ & $p$ & $\eta^{2}$ & $\mathrm{~F}$ & $p$ & $\eta^{2}$ & $\mathbf{F}$ & $\mathrm{p}$ & $\eta^{2}$ \\
\hline Task & 3.631 & 0.061 & .054 & 9.694 & 0.003 & .133 & & & & 10.680 & 0.002 & .145 & & & \\
\hline Load & & & & & & & & & & & & & 4.781 & 0.033 & .071 \\
\hline Site & 14.24 & $<0.001$ & .184 & 29.38 & $<0.001$ & .318 & 25.39 & $<0.001$ & .287 & 16.747 & $<0.001$ & .210 & 22.75 & $<0.001$ & .265 \\
\hline Hemisphere & 7.712 & 0.007 & .109 & & & & & & & & & & & & \\
\hline Group & & & & & & & 6.143 & 0.016 & .089 & & & & & & \\
\hline Site $\times$ Hemisphere & & & & & & & & & & 5.159 & 0.027 & .076 & & & \\
\hline Task $\times$ Group & & & & 4.763 & 0.033 & .070 & & & & & & & & & \\
\hline Task $\times$ Load & 4.415 & 0.040 & .065 & & & & & & & 6.376 & 0.014 & .092 & & & \\
\hline Task $\times$ Site & 8.285 & 0.005 & .116 & 5.620 & 0.021 & .082 & & & & & & & & & \\
\hline Task $\times$ Hemisphere & 4.270 & 0.043 & .063 & & & & & & & & & & & & \\
\hline Task $\times$ Site $\times$ Group & & & & & & & & & & & & & 5.194 & 0.026 & .076 \\
\hline Load $\times$ Site $\times$ Hemisphere & 7.586 & 0.008 & .107 & 5.363 & 0.024 & .078 & & & & & & & & & \\
\hline Task $\times$ Site $\times$ Hemisphere $\times$ Group & 9.042 & 0.004 & .126 & & & & & & & 5.131 & 0.027 & .075 & & & \\
\hline
\end{tabular}

Italic numbers indicate significant effects $(p<0.05)$

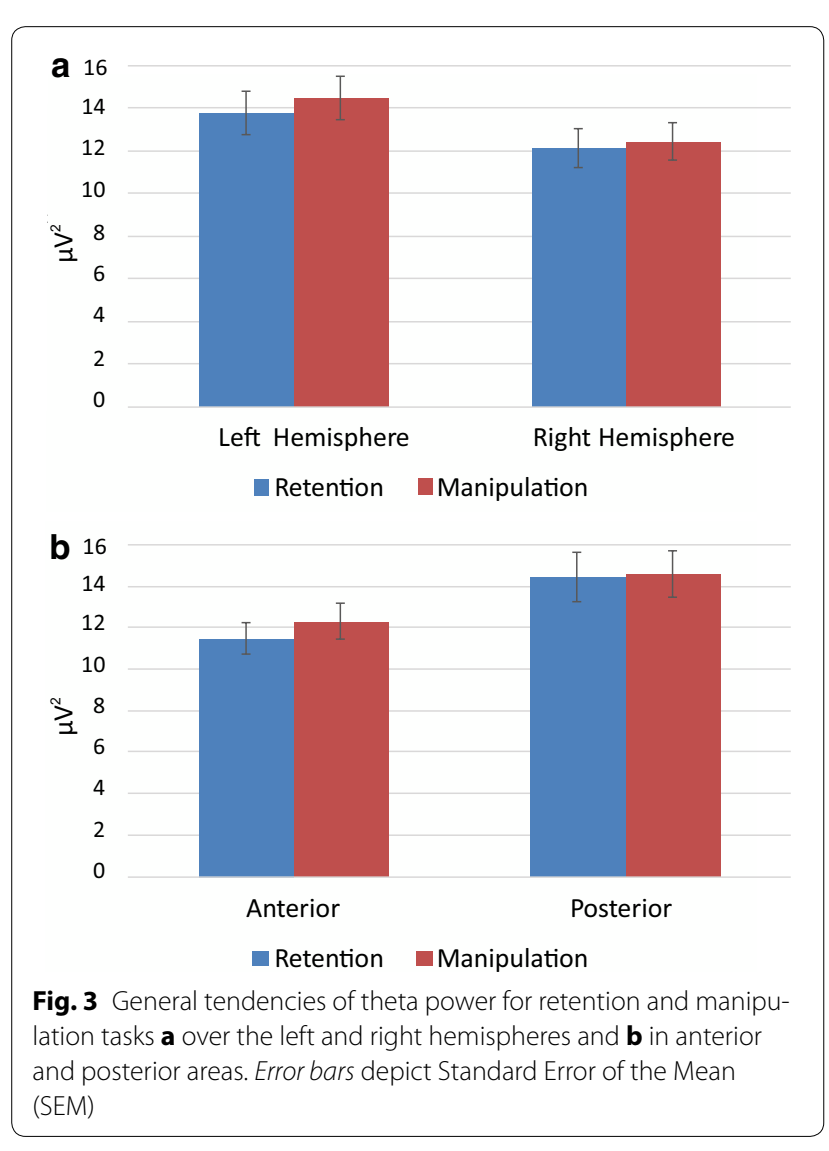

group we observed a larger theta power in the manipulation condition than in the retention condition, and the magnitude of this effect was the highest in the left anterior area (Task $\times$ Site $\times$ Hemisphere interaction within the HP group: $\left.\mathrm{F}(1,31)=7.605, \mathrm{p}=0.01, \eta^{2}=.197\right)$. No significant effects were found in the LP group.

The analysis of midline theta also showed a higher power in the manipulation task than in the retention task [main effect of Task, $\mathrm{F}(1,63)=7.685, \mathrm{p}=0.007$, $\left.\eta^{2}=.109\right]$. Increasing number of the presented letters from 5 to 7 yielded a decrease of theta power in the manipulation task but its increase in the retention task [Task $\times$ Load interaction, $F(1,63)=5.462, p=0.023$, $\left.\eta^{2}=.080\right]$. This interaction was, however, strongly modified by the between-subject factor. As depicted in Fig. 4, an increase of the number of letters from 5 to 7 was associated with an increase of theta activity in the HP group but its decrease in the LP group [Load $\times$ Group interaction, $F(1,63)=4.465, \mathrm{p}=0.039, \eta^{2}=.066$ ]. Figure 4 shows that the significant Load $\times$ Task interaction for the entire sample described above was actually produced by the dramatic decrement of the theta power in the most demanding condition (manipulation task, high WM load) in the LP group. Similarly, the triple interaction Load $\times$ Site $\times$ Hemisphere for the entire sample does not really characterize the entire sample but, like the Load $\times$ Task interaction, can be attributed to a disproportionately strong influence of the LP group.

\section{Alpha}

As expected, alpha1 and alpha 2 activity increased in the posterior direction (main effect of Site, see Table 2).

Alpha1 power was lower in the manipulation task than in the retention tasks (main effect of Task). This effect was larger at the posterior than anterior sites (Task $\times$ Site interaction). In addition, as can be seen in Fig. 5, the 


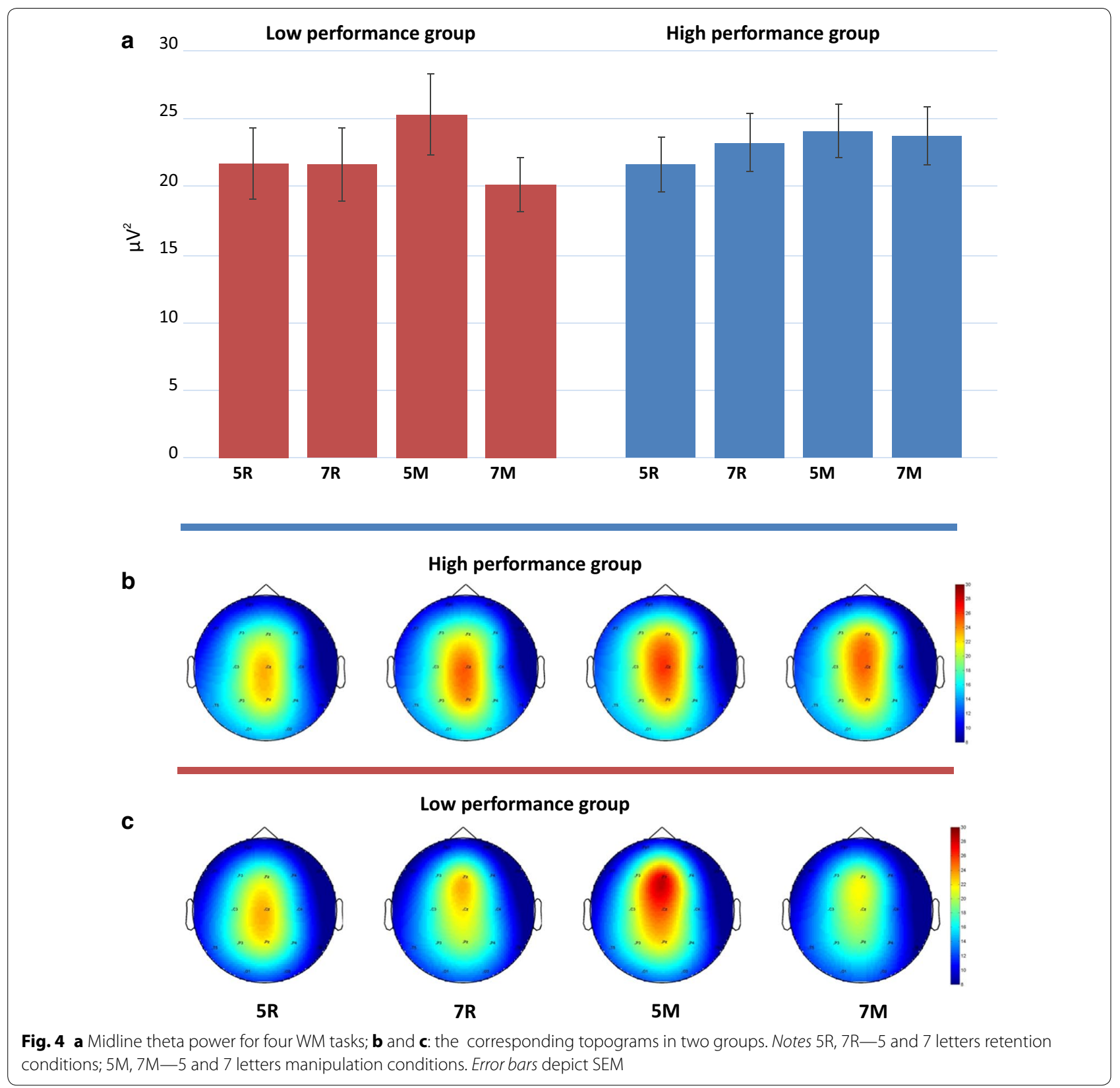

suppression of the alpha1 power in the manipulation task relative to the retention task was stronger in the HP than the LP group (Task $\times$ Group interaction). Alpha2 was generally stronger in the HP than the LP group (main effect of Group). There were no significant differences in the average individual alpha frequencies between the groups $[F(1,63)=0.14, p=0.71]$, with mean values being $10.47 \mathrm{~Hz}(\mathrm{SD}=0.87)$ and $10.39 \mathrm{~Hz}(\mathrm{SD}=0.82)$ in the $\mathrm{LP}$ and the HP groups, respectively.

Alpha1 activity was suppressed with increasing WM load in each ROI except the right posterior area where alpha1 power increased (Load $\times$ Site $\times$ Hemisphere interaction).

\section{Beta1}

Beta1 power was significantly lower in the anterior than posterior areas (main effect of Site), and lower on the left than right side (main effect of Hemisphere).

As can be seen in Fig. 6, beta1 power increased with the increasing WM load in the manipulation conditions but decreased in the retention conditions (Task $\times$ Load interaction). In general, the power was higher in the 


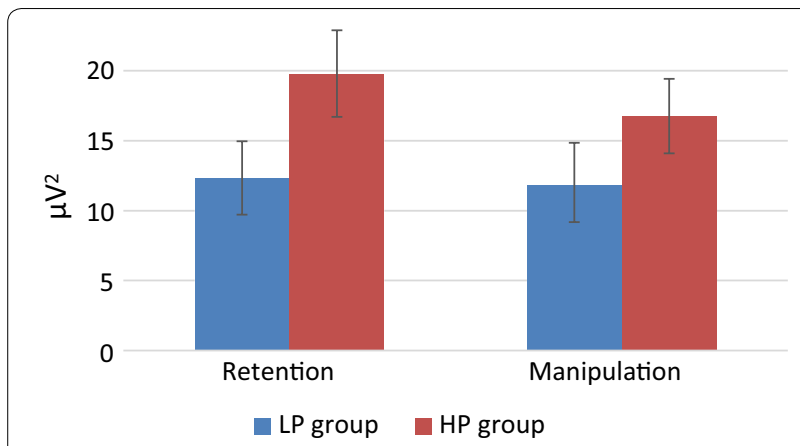

Fig. 5 Alpha1 power for retention and manipulation tasks in low and high performance groups. Error bars depict SEM

retention condition than in the manipulation condition (main effect of Task).

A significant four-way Task $\times$ Site $\times$ Hemisphere $\times$ Group interaction was obtained and further analyzed for groups and for electrode sites. The first ANOVA yielded a significant Task $\times$ Site $\times$ Hemisphere interaction $\left[\mathrm{F}(1,31)=6.471, \mathrm{p}<0.05, \eta^{2}=.131\right]$ only in the HP group, indicating that the decrease of the betal power from the retention task to the manipulation task was more pronounced in the left posterior and the right anterior ROIs. No such effects were observed in the LP group.

The second ANOVA revealed a significant Task $\times$ Group interaction in the left posterior ROI $\left[\mathrm{F}(1,31)=5953, \mathrm{p}<0.05, \eta^{2}=.086\right]$. This result converges with the preceding one, both indicating task dependent changes of beta1 power in the HP group only, and particularly at the left posterior area (see Fig. 7).

\section{Beta2}

In strike contrast to beta1, beta 2 power was significantly larger in the anterior than posterior areas (main effect of Site). Increasing WM load led to an increase in beta2 activity (main effect of Load).

The significant Task $\times$ Site $\times$ Group interaction (see Table 2) indicates opposite task- and location-related changes in the two groups. The HP group showed higher beta2 activity in the manipulation task at anterior areas, but in the retention task at posterior areas. The opposite held true for the LP group (Fig. 8).

\section{Discussion}

\section{Theta and central executive components of WM}

The current study found that increasing WM task complexity and executive control demand were associated with the increase of the frontal theta activity. Increasing theta power in midline and frontal areas during mental manipulations in contrast to the mere retention of memory content is in line with numerous data indicating positive relationships between FMT and cognitive load [17, 18, 42, 45-47]. Moreover, an increase of FMT in manipulation tasks as compared with retention tasks was also found in studies whose design was similar to the present one [21-23, 48].

In addition, the link between FMT and the activation of the anterior cingulate cortex (ACC) and the medial

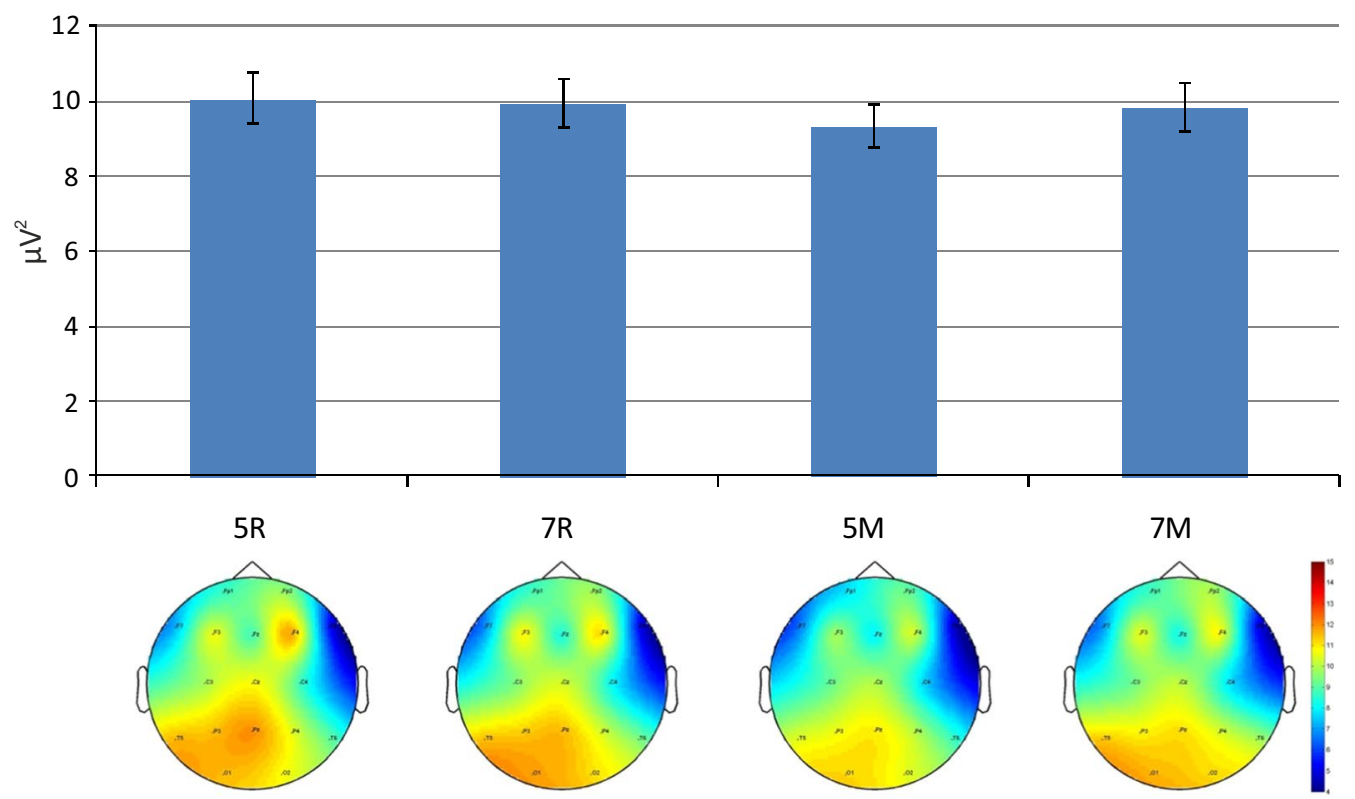

Fig. 6 Beta1 power chart and corresponding topograms for retention and manipulation tasks. Error bars depict SEM 


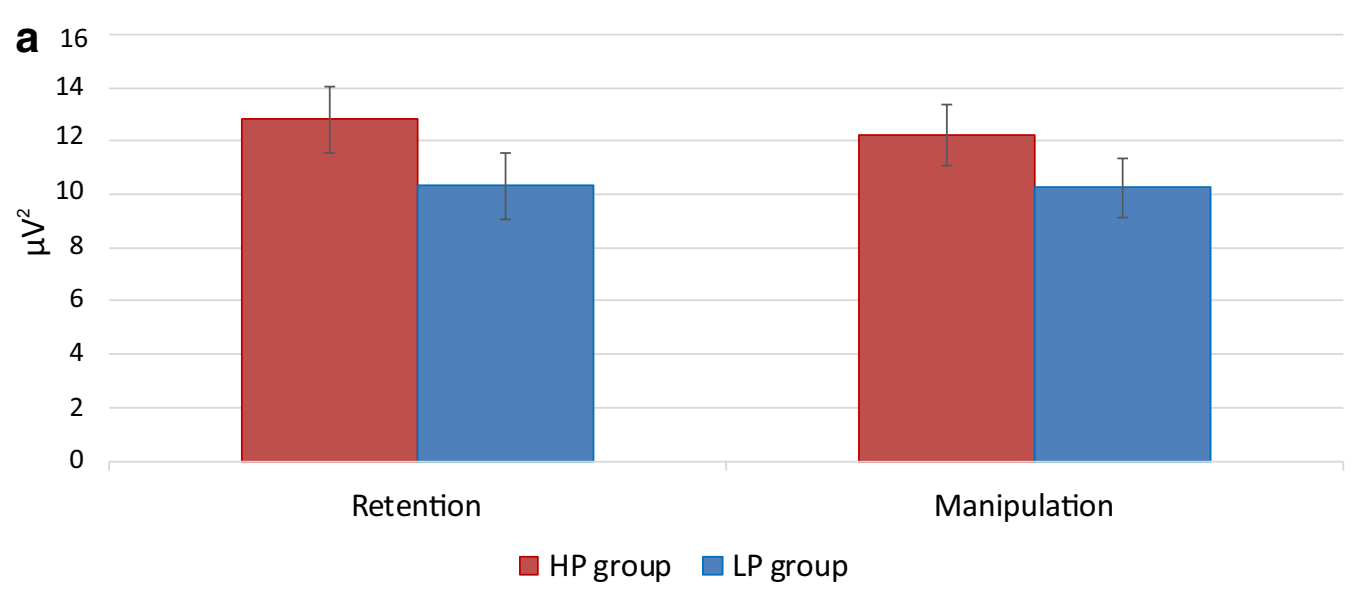

b

High performance group
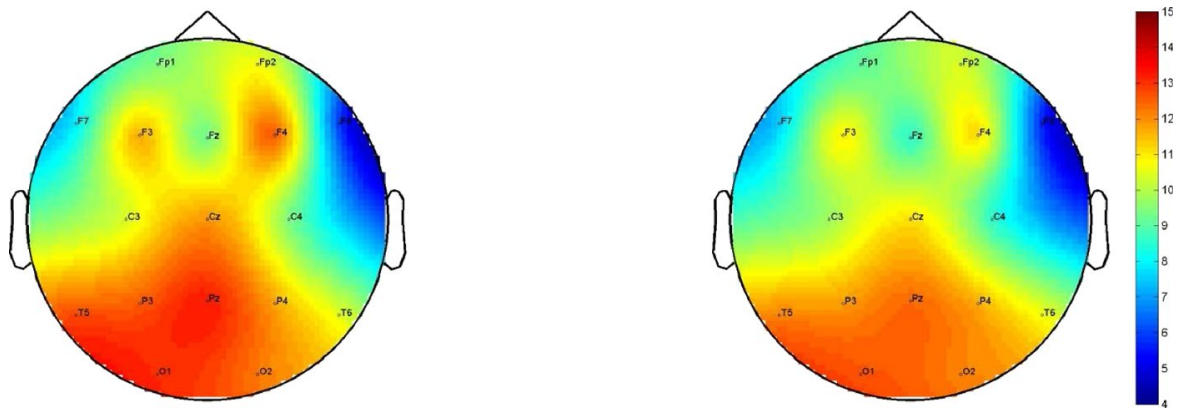

C

\section{Low performance group}

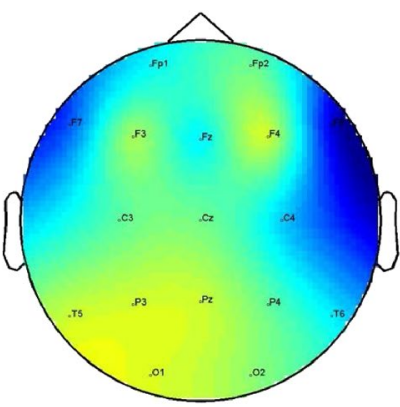

Retention

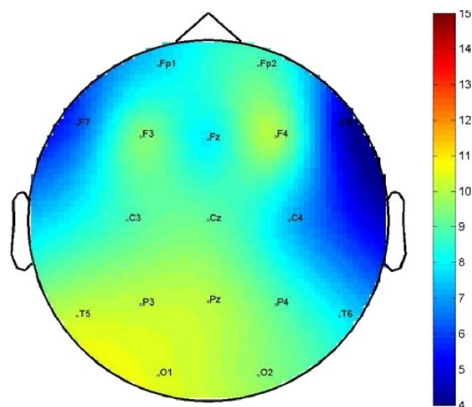

Manipulation

Fig. 7 Beta1 power in the left posterior area for retention and manipulation tasks (a) and corresponding topograms in Low performance (LP; b) and High performance (HP; c) groups. Error bars depict SEM

prefrontal cortex (mPFC) was repeatedly proven by simultaneous EEG-fMRI recordings as well as by direct electrophysiological recordings in monkeys [49-52]. The ACC and the mPFC are active during memory processes, WM performance, and executive control [53-55].
We assume that the increment of FMT (supposedly indicating the activation of the ACC) with increasing WM demands is related to increasing involvement of executive processes. However, it should be noted that FMT reflects not pure memory processes per se but more likely the allocation of cortical resources depending on 

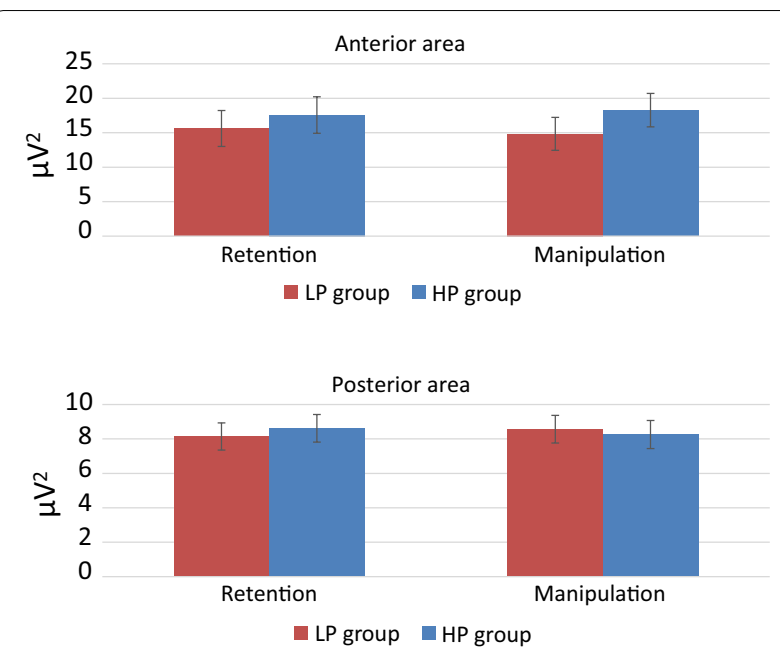

Fig. 8 Beta2 power for Retention and Manipulation tasks in low and high performance groups in anterior and posterior areas. Error bars depict SEM

the features of the task $[18,55,56]$. One may speculate that increasing demands for executive control during manipulation of information in WM engage a widely distributed network whose main components are the prefrontal cortex and the ACC.

The task-related increment of the theta power in the left anterior area was found only in the HP group. This may be related to more effective manipulations supported by the language cortex. Some authors hypothesize that high WM load leads to the involvement of a circuit including the prefrontal cortex and the medial temporal lobe related to long-term memory [57-59]. The activation of the left prefrontal cortex including the inferior frontal gyrus (IFG) and Broca's area was found in verbal tasks associated with executive functions [51, 60, 61]. Simultaneous EEG/fMRI recording in a modified Sternberg task revealed a load-dependent increase of left IFG activation and the theta rhythm [51]. Similar results were obtained by Chee and Choo [62] in another WM task. We suppose that the left-hemispheric accentuation of the theta rhythm may represent more effective information exchange between short- and long-term memory storage in the HP group.

Group differences were not only task-dependent but also load-dependent. The HP group demonstrated a gradual increase of theta power at midline, reaching its peak in the most demanding condition: manipulation task with 7 letters. In contrast, the LP group exhibited a sharp drop of theta power in this condition after a maximum in the condition of moderate difficulty: manipulation with 5 letters. Since previous studies of EEG correlates of individual differences in WM were limited to moderate difficulty, we can state that our findings are fully consistent with the previous ones, where the theta activity always increased with memory load $[18,40,42$, $43,45,46]$. However, the most difficult task resulted in a more complex change of theta activity that has not been observed so far.

One may speculate that reaching the individual's WM capacity limit is accompanied by a crucial deficit of attentional resources. Post-experimental reports suggest that most participants formulated their task as "to remember all letters if possible", but possibly, some LP participants in the most difficult condition changed the task to "to remember at least some letters". Alternatively, some subjects may have switched strategy to "remember the first few letters with regard to position" in the forward task and the "first few letters with regard to alphabetical order" in the alphabetical task. This post hoc hypothesis was supported by an analysis of behavioral results regarding to the position of the probe letter. The factor Position was taken with 2 levels (the first two vs. the last 2 letters for 5-letters conditions, or the first three vs. the last 3 letters for 7-letters conditions). Both 2-way Position $\times$ Group interaction $[\mathrm{F}(1,63)=6.022, \mathrm{p}=0.017$, $\left.\eta^{2}=.087\right]$ and a 4-way Position $\times$ Task $\times$ Load $\times$ Group interaction $\left[\mathrm{F}(1,63)=3.183, \mathrm{p}=0.045, \eta^{2}=.048\right]$ were significant. Unfortunately, due to the post hoc nature of this effect we could not perform the EEG analysis with the factor Position, because we did not have a sufficient statistical power for this unplanned comparison.

Another explanation might be the loss of motivation in LP participants in the most challenging condition. This hypothesis, however, would predict a particularly poor performance of LP participants in the manipulation task with 7 letters. This disagrees with the observed data indicating nearly equal performance differences between LP and HP participants in all conditions (see Fig. 4). From our point of view, the strategy change hypothesis can better integrate this fact that the loss-of-motivation hypothesis.

Also Jaeggi et al. [41] came on the basis of their fMRI study to the same conclusion concerning the suboptimal strategies used by LP subjects in WM tasks. In that study, LP participants showed a positive correlation between task complexity and the amount of the broad activation in the frontal cortex. Obviously, the most challenging condition leads to the widely distributed engagement of the prefrontal cortex and results in the lack of neural resources for activation of the ACC necessary for the executive control of WM.

\section{Alpha and the storage components of WM}

As compared with the retention condition, manipulation of stimuli in WM was associated with distributed 
suppression of alpha1 activity. Desynchronization of low alpha has been regarded as a nonspecific cortical response that can be observed during various cognitive operations $[28,42,63]$ including maintaining information in WM $[49,64,65]$. In addition to this non-specificity model, however, more specific hypotheses about the dynamics of alpha exist. Thus, alpha synchronization in posterior areas during the maintenance of actual information may reflect active inhibition to protect these areas from reorienting to new irrelevant information processing $[17,32]$. It is plausible that the temporary storage components of WM play a key role in successful maintenance of 7 letters relative to 5 letters. It might be suggested that when the volume of information maintained in the temporary storage approaches the putative capacity limit $(7 \pm 2)$, the central executive should actively inhibit irrelevant information. The observed asymmetry of alpha1 power at the posterior area agrees with the previous studies of WM and short-term memory [17, 42, 50, $66,67]$.

In the development of the cortical idling hypothesis, Pfurtscheller et al. [68] proposed that the increasing alpha activity during cognitive processing is related to the allocation of attentional resources by inhibition of the cortical areas irrelevant to the current task [32, 69, 70]. In this context, the alpha rhythm plays a role of an information flow filter.

It is well known that WM is one of the main components of general intelligence [71, 72]. Accordingly, the degree of alpha desynchronization in semantic memory task is positively related to intelligence [73]. Similar correlations between IQ and alpha power were observed in the resting state $[74,75]$. We suppose that stronger alpha power may reflect a higher level of readiness to perceive relevant information. Therefore, HP individuals have potentially more resourceful visual cortex and manage the tasks better [63].

\section{Beta1 and manipulation of information in WM}

Task-related decrement of beta1 power found in this study was quite similar to the effect reported by Berger et al. [22] who also compared manipulation versus retention conditions. This effect may be explained by the conception of Engel and Fries [76] that, applied to the present experiment, suggests that the decrease of beta1 power takes place during updating or manipulating information in WM as well as during retrieval of information from long term memory and encoding it in WM. The desynchronization of the betal rhythm can be attributed to the sequential updating of the WM content during mental alphabetizing of the letters. This process also involves addressing the long term memory where the alphabet is stored.
Load-dependent changes in beta1 power were observed only in the manipulation condition. We hypothesize that manipulation is performed using two independent temporal buffers. One of them ("store" buffer) is the final storage for modified items after the manipulations, and the other one ("working" buffer) serves as a workspace for the remaining to-be-modified items. Weiss and Müller [77] hypothesize about two different beta1 rhythms that overlap in frequency but reflect different subprocesses in WM. The first rhythm supports the activity of the "store" buffer, and the second rhythm, that of the "workspace" buffer. Synchronization of the former maintains the active state of the engram and protects it from irrelevant information. Weiss and Rappelsberger [78] demonstrated a gradual increase of betal activity in response to sequential filling of WM by words. Research conducted by Leiberg et al. [79] also showed a loaddependent increase of betal activity. At the same time, desynchronization of the other beta1 rhythm reflects the retrieval from long term memory and encoding to WM. In other words, desynchronization of the latter beta1 rhythm reflects manipulations of objects in the "workspace" for their subsequent transfer to the "store".

Our hypothesis also entails that the lack of beta1 desynchronization during the encoding process indicates a disruption of memory formation. Recently, Hanslmayr et al. [80] found a negative effect of transcranial magnetic stimulation (TMS) of the left inferior frontal gyrus at beta1 frequency $(18.7 \mathrm{~Hz})$ on memory performance in a word-list learning task. Furthermore, a study [34] performed on monkeys demonstrated desynchronization of beta activity during updating of WM content but synchronization of beta activity during retention.

Probably, in the retention condition the workspace buffer is only minimally involved. It may work at the beginning of the delay period when sequentially and quickly presented information is encoded. Thus Zanto and Gazzaley [81] found the desynchronization of beta1 rhythm during the first $1250 \mathrm{~ms}$ of the 4-s delay period but its synchronization from $1500 \mathrm{~ms}$ to the end of the delay. In the current study, the delay periods during maintaining and manipulation of 5 and 7 letters could be different due to a longer presentation time ( $3 \mathrm{~s})$. Therefore, the recombination of 5 letters to the alphabetical order could already start during stimulus presentation and continue only in the workspace buffer without addressing the store buffer. When the recombination process is finished, the result is transferred to the store buffer and kept there until the probe is presented. The store buffer in this case prevents possible interference of other stimuli and maintains the actual state of the engram until the moment when its content is requested. When a longer stimulus set is memorized (i.e., 7 letters), a plausible strategy is to 
memorize the initial letters set and to transfer it into the store buffer. If this strategy is used, recombination may start after the stimuli have disappeared from the screen. During this period, both buffers are actively involved: the store buffer is keeping the initial set, while recombination is carried out in the workspace buffer. When the recombination is finished, the information moves to the store and updates its content. This assumed information return to, and updating of, the store buffer would explain the increase of beta 1 power from 5- to 7-letter condition in the manipulation task.

At the level of individual differences, the main result was a stronger desynchronization of beta1 rhythm in the HP group during manipulation condition in the left posterior area. An important role of the superior parietal cortex in flexible redistribution of attentional resources was demonstrated in several studies [82-85]. In terms of the proposed two-buffer model, one may suggest that HP individuals are better able to shift their attention between the store of the originally presented set and the workspace where they work with the symbols. This might allow them to perform manipulations in the "workspace" buffer not spending too much resources for maintaining information in the "store" buffer.

\section{Beta2 and amount of information in WM}

Beta2 power increased with the increasing WM load and did not significantly depend on the type of the task.

Dissociations between the lower $(13-20 \mathrm{~Hz})$ and upper $(25-30 \mathrm{~Hz})$ beta were demonstrated earlier in a study of Shahin et al. [86]. The authors concluded that the increment of the upper beta may reflect maintaining verbal stimuli in auditory memory. The maintenance of stimuli in WM was also suggested to cause synchronization of beta2 $(\sim 20-30 \mathrm{~Hz})$ in two different tasks $[87,88]$. Spitzer et al. [88] assumed that the upper beta activity is directly related to the quantity of supramodal abstract information. The significant effect of Load on beta2 power found in the present study is in line with this interpretation.

During the manipulation task, beta2 power increased in the HP group in the anterior areas, but in the LP group in the posterior areas. As we do not know any comparable result in the literature, this finding is, first of all, in need of replication. As a putative explanation, we propose that beta 2 may most simply be designated as "activation". We believe, therefore, that changes in beta2 activity are not related to mental processes as such, but rather to the general volume of information necessarily used in these processes. This volume is expected to be larger in the manipulation task than in the retention task because during manipulation one has to work with at least two stimulus sets: the one that should be manipulated with and the one that results from the manipulation. The increase of frontal activity in HP participants may, therefore, reflect their ability to process a larger amount of information, whereas the heightened activity of sensory regions in LP subjects appears to reflect their need to frequently address the original stimulus set.

\section{Summary of the proposed model}

In general, the obtained results allow us to make several claims about possible factors contributing, at the individual level, to effective verbal WM performance:

firstly, a higher state of readiness to process relevant and to inhibit irrelevant information and related larger alpha power;

secondly, stronger engagement of the left prefrontal cortex; this factor can underlie efficient maintaining and manipulating information in WM due to a fast exchange of information between long term and working memory;

thirdly, an energy efficient strategy for distribution of frontal resources in order to maintain the necessary level of activity of the ACC;

finally, activation of the ACC and the related executive functions is decisive for successful manipulations of content in WM, simultaneous maintaining information about initial properties of stimuli and efficiently shifting attention between these cognitive operations.

\section{Limitations}

We have to acknowledge at least two limitations of the present study. Firstly, the results may be affected by the homogeneity of the sample in respect to gender (i.e., females). A gender based analysis will be the matter of a subsequent report. Secondly, our putative explanation hypotheses suggested in the Discussion above have neuroanatomical implications, i.e., they presume the activity of certain brain structures such as the ACC. To test these hypotheses, a larger number of electrodes should be used in future studies, which will allow a more precise assessment of the spatial distribution of the obtained effects. Thirdly, individual differences were approached using median split on the global performance. Although the data show that general performance differences play a significant role, this approach may underestimate the importance the individual differences that concern only specific conditions (e.g., only manipulation but not retention). In principle, other approaches to group selection are possible and might yield different results. 


\section{Conclusions}

1. In accordance with many previous studies, we expected to find significant WM-related changes in alpha and theta frequency bands. This hypothesis was only partially supported by the data. Significant effects were found in all analyzed frequency bands from theta to high beta, indicating that our knowledge about the neural basis of WM is not comprehensive.

2. The hypothesis about a strong participation of the frontal theta rhythm in WM processes was confirmed. The novel finding was, however, different dynamics of frontal theta in HP and LP groups.

3. When starting the study, we believed that some important findings can have been missed in the previous experiments because they used only tasks of low to average difficulty. Therefore, we predicted important intergroup variation in EEG pattern in the most challenging condition. This prediction was confirmed. The most pronounced differences between individuals with high and low WM performance, in terms of the oscillatory activity in several frequency ranges, were observed in the manipulation task with 7 letters, which is a very difficult condition that for many individuals might exceed their limits. Particularly, this condition resulted in a more complex change of theta activity than just an increase with WM load, which has not been observed so far. Including greater variety of experimental conditions and groups to the WM research agenda seems beneficial.

4. Finally, we expected a stronger effect of executive WM components as compared with storage components. The data put this hypothesis in question. Firstly, the difference in performance between LP and HP participants was nearly equal in retention (weak executive control demands) and manipulation (much higher executive control demands) conditions. Secondly, task and site dependent group differences were found in each explored frequency bands including anterior theta and posterior alpha activity. In some studies these two responses were interpreted as reflections of executive and storage components of WM, respectively $[89,90]$. Although there is an alternative interpretation on the basis of cross-frequency coupling [91, 92], all these observations together may indicate that the two components of WM are equally important for WM performance at the individual level. More studies are needed to clarify this issue.

\section{Abbreviations}

WM: working memory; HP: high performance; LP: low performance; tACS: transcranial alternating current stimulation; FMT: frontal midline theta rhythm;
EEG: electroencephalography; EOG: electrooculography; IAF: individual alpha frequency; FFT: fast Fourier transformation; ICA: independent component analysis; ROI: region of interest; SEM: standard error of the mean; IFG: inferior frontal gyrus; fMRI: functional magnetic resonance imaging; ACC: anterior cingulate cortex; IQ: intelligence quotient; ANOVA: analysis of variance.

\section{Authors' contributions}

YGP conceived of the study, designed the experimental paradigm, carried out the recording of the data, performed the statistical analysis and drafted the manuscript. BK contributed to the discussion, and to the preparation of the manuscript. Both authors read and approved the final manuscript.

\section{Author details \\ ${ }^{1}$ Institute of Medical Psychology and Behavioural Neurobiology, University of Tübingen, Tübingen, Germany. ${ }^{2}$ Department of Psychology, Ural Federal University, Yekaterinburg, Russia.}

\section{Competing interests}

The authors declare that they have no competing interests.

\section{Availability of data and materials}

The datasets analyzed during the current study is available from the corresponding author on reasonable request.

\section{Ethics approval and consent to participate}

Informed consent was obtained from all subjects prior to the study. The study was approved by the Ural Federal University Ethics Committee.

Funding

We acknowledge support by the Deutsche Forschungsgemeinschaft and Open Access Publishing Fund of University of Tübingen.

Received: 23 December 2016 Accepted: 4 February 2017

Published online: 13 February 2017

\section{References}

1. Daneman M, Merikle PM. Working memory and language comprehension: a meta-analysis. Psychon Bull Rev. 1996;3:422-33.

2. D'Esposito M. From cognitive to neural models of working memory. Philos Trans R Soc B Biol Sci. 2007:362:761-72.

3. Hyun J-S, Luck SJ. Visual working memory as the substrate for mental rotation. Psychon Bull Rev. 2007:14:154-8.

4. Baddeley A. Working memory: theories, models, and controversies. Annu Rev Psychol. 2012:63:1-29.

5. Baddeley A. Working memory: looking back and looking forward. Nat Rev Neurosci. 2003:4:829-39.

6. Engle RW, Tuholski SW, Laughlin JE, Conway AR. Working memory, shortterm memory, and general fluid intelligence: a latent-variable approach. J Exp Psychol Gen. 1999;128:309.

7. Postle BR. Working memory as an emergent property of the mind and brain. Neuroscience. 2006:139:23-38.

8. Nee DE, Brown JW, Askren MK, Berman MG, Demiralp E, Krawitz A, et al. A meta-analysis of executive components of working memory. Cereb Cortex. 2013;23:264-82

9. Cohen JD, Perlstein WM, Braver TS, Nystrom LE, Noll DC, Jonides J, et al Temporal dynamics of brain activation during a working memory task. Nature. 1997:386:604-8.

10. Edin F, Klingberg T, Johansson P, McNab F, Tegnér J, Compte A. Mechanism for top-down control of working memory capacity. Proc Natl Acad Sci. 2009;106:6802-7.

11. Klingberg T, O'Sullivan BT, Roland PE. Bilateral activation of fronto-parietal networks by incrementing demand in a working memory task. Cereb Cortex. 1997;7:465-71.

12. Linden DEJ, Bittner RA, Muckli L, Waltz JA, Kriegeskorte N, Goebel R, et al. Cortical capacity constraints for visual working memory: dissociation of fMRI load effects in a fronto-parietal network. Neurolmage. 2003;20:1518-30 
13. Palva JM, Monto S, Kulashekhar S, Palva S. Neuronal synchrony reveals working memory networks and predicts individual memory capacity. Proc Natl Acad Sci. 2010;107:7580-5.

14. Ekman M, Fiebach CJ, Melzer C, Tittgemeyer M, Derrfuss J. Different roles of direct and indirect frontoparietal pathways for individual working memory capacity. J Neurosci. 2016;36:2894-903.

15. Buzsaki G. Neuronal oscillations in cortical networks. Science. 2004;304:1926-9.

16. Aftanas LI, Golocheikine SA. Human anterior and frontal midline theta and lower alpha reflect emotionally positive state and internalized attention: high-resolution EEG investigation of meditation. Neurosci Lett. 2001;310:57-60

17. Jensen $O$, Tesche $C D$. Frontal theta activity in humans increases with memory load in a working memory task. Eur J Neurosci. 2002;15:1395-9.

18. Onton J, Delorme A, Makeig S. Frontal midline EEG dynamics during working memory. Neurolmage. 2005;27:341-56.

19. Sauseng P, Klimesch W, Schabus M, Doppelmayr M. Fronto-parietal EEG coherence in theta and upper alpha reflect central executive functions of working memory. Int J Psychophysiol. 2005;57:97-103.

20. Sauseng P, Griesmayr B, Freunberger R, Klimesch W. Control mechanisms in working memory: a possible function of EEG theta oscillations. Neurosci Biobehav Rev. 2010:34:1015-22.

21. Berger B, Minarik T, Griesmayr B, Stelzig-Schoeler R, Aichhorn W, Sauseng P. Brain oscillatory correlates of altered executive functioning in positive and negative symptomatic schizophrenia patients and healthy controls. Front Psychol. 2016;7:705

22. Berger B, Omer S, Minarik T, Sterr A, Sauseng P. Interacting memory systems-does EEG alpha activity respond to semantic long-term memory access in a working memory task? Biology. 2014;4:1-16.

23. Griesmayr B, Gruber WR, Klimesch W, Sauseng P. Human frontal midline theta and its synchronization to gamma during a verbal delayed match to sample task. Neurobiol Learn Mem. 2010;93:208-15.

24. Cooper PS, Wong ASW, Fulham WR, Thienel R, Mansfield E, Michie PT, et al. Theta frontoparietal connectivity associated with proactive and reactive cognitive control processes. Neurolmage. 2015;108:354-63.

25. Sarnthein J, Petsche H, Rappelsberger P, Shaw GL, Von Stein A. Synchronization between prefrontal and posterior association cortex during human working memory. Proc Natl Acad Sci. 1998;95:7092-6.

26. Wu X, Chen X, Li Z, Han S, Zhang D. Binding of verbal and spatial information in human working memory involves large-scale neural synchronization at theta frequency. Neurolmage. 2007;35:1654-62.

27. Polanía R, Nitsche MA, Korman C, Batsikadze G, Paulus W. The importance of timing in segregated theta phase-coupling for cognitive performance. Curr Biol. 2012:22:1314-8.

28. Klimesch W. Alpha-band oscillations, attention, and controlled access to stored information. Trends Cogn Sci. 2012;16:606-17.

29. Sauseng P, Klimesch W, Heise KF, Gruber WR, Holz E, Karim AA, et al. Brain oscillatory substrates of visual short-term memory capacity. Curr Biol. 2009:19:1846-52.

30. Tuladhar AM, ter Huurne N, Schoffelen J-M, Maris E, Oostenveld R, Jensen O. Parieto-occipital sources account for the increase in alpha activity with working memory load. Hum Brain Mapp. 2007;28:785-92.

31. Jensen O, Mazaheri A. Shaping functional architecture by oscillatory alpha activity: gating by inhibition. Front Hum Neurosci [Internet]. 2010 [cited 2016 May 23];4. http://journal.frontiersin.org/article/10.3389/ fnhum.2010.00186/abstract.

32. Klimesch W, Sauseng P, HansImayr S. EEG alpha oscillations: the inhibition-timing hypothesis. Brain Res Rev. 2007;53:63-88.

33. Deiber M-P, Missonnier P, Bertrand O, Gold G, Fazio-Costa L, Ibanez V, et al. Distinction between perceptual and attentional processing in working memory tasks: a study of phase-locked and induced oscillatory brain dynamics. Cogn Neurosci J. 2007;19:158-72.

34. Siegel M, Warden MR, Miller EK. Phase-dependent neuronal coding of objects in short-term memory. Proc Natl Acad Sci. 2009;106:21341-6.

35. Tallon-Baudry C, Bertrand O, Peronnet F, Pernier J. Induced $\gamma$-band activity during the delay of a visual short-term memory task in humans. J Neurosci. 1998;18:4244-54.

36. Barrett LF, Tugade MM, Engle RW. Individual differences in working memory capacity and dual-process theories of the mind. Psychol Bull. 2004;130:553-73.
37. Engle RW, Kane MJ. Executive attention, working memory capacity, and a two-factor theory of cognitive control. Psychol Learn Motiv. 2004:44:145-200.

38. Unsworth N, Spillers GJ. Working memory capacity: attention control, secondary memory, or both? A direct test of the dual-component model. J Mem Lang. 2010;62:392-406.

39. Brouwer A-M, Hogervorst MA, van Erp JBF, Heffelaar T, Zimmerman PH, Oostenveld R. Estimating workload using EEG spectral power and ERPs in the n-back task. J Neural Eng. 2012;9:45008.

40. Daffner KR, Chong H, Sun X, Tarbi EC, Riis JL, McGinnis SM, et al. Mechanisms underlying age- and performance-related differences in working memory. J Cogn Neurosci. 2011;23:1298-314.

41. Jaeggi SM, Buschkuehl M, Etienne A, Ozdoba C, Perrig WJ, Nirkko AC. On how high performers keep cool brains in situations of cognitive overload. Cogn Affect Behav Neurosci. 2007;7:75-89.

42. Gevins A, Smith ME, McEvoy L, Yu D. High-resolution EEG mapping of cortical activation related to working memory: effects of task difficulty, type of processing, and practice. Cereb Cortex. 1997:7:374-85.

43. Pesonen $\mathrm{M}$, Hämäläinen $\mathrm{H}$, Krause $\mathrm{CM}$. Brain oscillatory $4-30 \mathrm{~Hz}$ responses during a visual n-back memory task with varying memory load. Brain Res. 2007;1138:171-7.

44. Dong S, Reder LM, Yao Y, Liu Y, Chen F. Individual differences in working memory capacity are reflected in different ERP and EEG patterns to task difficulty. Brain Res. 2015;1616:146-56.

45. Itthipuripat $S$, Wessel JR, Aron AR. Frontal theta is a signature of successful working memory manipulation. Exp Brain Res. 2012;224:255-62.

46. Missonnier P, Deiber M-P, Gold G, Millet P, Pun MG-F, Fazio-Costa L, et al. Frontal theta event-related synchronization: comparison of directed attention and working memory load effects. J Neural Transm. 2006;113:1477-86.

47. Gevins A, Smith ME. neurophysiological measures of working memory and individual differences in cognitive ability and cognitive style. Cereb Cortex. 2000;10:829-39.

48. Griesmayr B, Berger B, Stelzig-Schoeler R, Aichhorn W, Bergmann J, Sauseng P. EEG theta phase coupling during executive control of visual working memory investigated in individuals with schizophrenia and in healthy controls. Cogn Affect Behav Neurosci. 2014;14:1340-55.

49. Meltzer JA, Negishi M, Mayes LC, Constable RT. Individual differences in EEG theta and alpha dynamics during working memory correlate with fMRI responses across subjects. Clin Neurophysiol. 2007:118:2419-36.

50. Michels $L$, Bucher K, Lüchinger R, Klaver P, Martin E, Jeanmonod D, et al. Simultaneous EEG-fMRI during a working memory task: modulations in low and high frequency bands. PLoS ONE. 2010;5:e10298.

51. Scheeringa R, Petersson KM, Oostenveld R, Norris DG, Hagoort P, Bastiaansen MCM. Trial-by-trial coupling between EEG and BOLD identifies networks related to alpha and theta EEG power increases during working memory maintenance. Neurolmage. 2009;44:1224-38.

52. Tsujimoto T, Shimazu H, Isomura Y, Sasaki K. Theta oscillations in primate prefrontal and anterior cingulate cortices in forewarned reaction time tasks. J Neurophysiol. 2010;103:827-43.

53. Bush G, Luu P, Posner Ml. Cognitive and emotional influences in anterior cingulate cortex. Trends Cogn Sci. 2000;4:215-22.

54. Niendam TA, Laird AR, Ray KL, Dean YM, Glahn DC, Carter CS. Meta-analytic evidence for a superordinate cognitive control network subserving diverse executive functions. Cogn Affect Behav Neurosci. 2012:12:241-68.

55. Shenhav A, Botvinick MM, Cohen JD. The expected value of control: an integrative theory of anterior cingulate cortex function. Neuron. 2013;79:217-40.

56. Sauseng P, Hoppe J, Klimesch W, Gerloff C, Hummel FC. Dissociation of sustained attention from central executive functions: local activity and interregional connectivity in the theta range. Eur J Neurosci. 2007:25:587-93.

57. Cashdollar N, Malecki U, Rugg-Gunn FJ, Duncan JS, Lavie N, Duzel E. Hippocampus-dependent and-independent theta-networks of active maintenance. Proc Natl Acad Sci. 2009;106:20493-8.

58. Eckart C, Fuentemilla L, Bauch EM, Bunzeck N. Dopaminergic stimulation facilitates working memory and differentially affects prefrontal low theta oscillations. Neurolmage. 2014:94:185-92.

59. Jeneson A, Squire LR. Working memory, long-term memory, and medial temporal lobe function. Learn Mem. 2011;19:15-25. 
60. Nelson J, Reuter-Lorenz PA, Persson J, Sylvester C-YC, Jonides J. Mapping interference resolution across task domains: a shared control process in left inferior frontal gyrus. Brain Res. 2009;1256:92-100.

61. Zhang JX, Feng C-M, Fox PT, Gao J-H, Tan LH. Is left inferior frontal gyrus a general mechanism for selection? Neurolmage. 2004;23:596-603.

62. Chee MWL, Choo WC. Functional imaging of working memory after $24 \mathrm{hr}$ of total sleep deprivation. J Neurosci. 2004;24:4560-7.

63. Klimesch W, Vogt F, Doppelmayr M. Interindividual differences in alpha and theta power reflect memory performance. Intelligence. 1999;27:347-62.

64. Maurer U, Brem S, Liechti M, Maurizio S, Michels L, Brandeis D. Frontal midline theta reflects individual task performance in a working memory task. Brain Topogr. 2015;28:127-34.

65. Michels L, Moazami-Goudarzi M, Jeanmonod D, Sarnthein J. EEG alpha distinguishes between cuneal and precuneal activation in working memory. Neurolmage. 2008;40:1296-310.

66. Babiloni C, Babiloni F, Carducci F, Cappa SF, Cincotti F, Del Percio C, et al. Human cortical rhythms during visual delayed choice reaction time tasks. Behav Brain Res. 2004;153:261-71.

67. Liu Z-X, Glizer D, Tannock R, Woltering S. EEG alpha power during maintenance of information in working memory in adults with ADHD and its plasticity due to working memory training: a randomized controlled trial. Clin Neurophysiol. 2016;127:1307-20.

68. Pfurtscheller G, Stancak A, Neuper C. Event-related synchronization (ERS) in the alpha band-an electrophysiological correlate of cortical idling: a review. Int J Psychophysiol. 1996;24:39-46.

69. Brunia CHM, Van Boxtel GJM. Wait and see. Int J Psychophysiol. 2001:43:59-75.

70. Pfurtscheller G, Neuper C, Flotzinger D, Pregenzer M. EEG-based discrimination between imagination of right and left hand movement. Electroencephalogr Clin Neurophysiol. 1997;103:642-51.

71. Conway AR, Cowan N, Bunting MF, Therriault DJ, Minkoff SR. A latent variable analysis of working memory capacity, short-term memory capacity, processing speed, and general fluid intelligence. Intelligence. 2002;30:163-83.

72. Kane MJ, Hambrick DZ, Conway ARA. Working memory capacity and fluid intelligence are strongly related constructs: comment on Ackerman, Beier, and Boyle (2005). Psychol Bull. 2005;131:66-71.

73. Doppelmayr M, Klimesch W, Hödlmoser K, Sauseng P, Gruber W. Intelligence related upper alpha desynchronization in a semantic memory task. Brain Res Bull. 2005;66:171-7.

74. Doppelmayr M, Klimesch W, Stadler W, Poellhuber D, Heine C. EEG alpha power and intelligence. Intelligence. 2002;30:289-302.

75. Jaušovec N. Differences in EEG alpha activity related to giftedness. Intelligence. 1996;23:159-73.

76. Engel AK, Fries P. Beta-band oscillations—signalling the status quo? Curr Opin Neurobiol. 2010;20:156-65.
77. Weiss S, Mueller HM. :Too many betas do not spoil the broth": the role of beta brain oscillations in language processing. Front Psychol [Internet]. 2012 [cited 2016 May 14];3. http://journal.frontiersin.org/article/10.3389/ fpsyg.2012.00201/abstract.

78. Weiss S, Rappelsberger P. Long-range EEG synchronization during word encoding correlates with successful memory performance. Cogn Brain Res. 2000;9:299-312.

79. Leiberg S, Lutzenberger W, Kaiser J. Effects of memory load on cortical oscillatory activity during auditory pattern working memory. Brain Res. 2006;1120:131-40.

80. Hanslmayr S, Matuschek J, Fellner M-C. Entrainment of prefrontal beta oscillations induces an endogenous echo and impairs memory formation. Curr Biol. 2014;24:904-9.

81. Zanto TP, Gazzaley A. Neural suppression of irrelevant information underlies optimal working memory performance. J Neurosci. 2009;29:3059-66.

82. Koenigs M, Barbey AK, Postle BR, Grafman J. Superior parietal cortex is critical for the manipulation of information in working memory. J Neurosci. 2009:29:14980-6.

83. Molenberghs P, Mesulam MM, Peeters R, Vandenberghe RRC. Remapping attentional priorities: differential contribution of superior parietal lobule and intraparietal sulcus. Cereb Cortex. 2007;17:2703-12.

84. Shomstein S, Yantis S. Control of attention shifts between vision and audition in human cortex. J Neurosci. 2004;24:10702-6.

85. Yantis S, Schwarzbach J, Serences JT, Carlson RL, Steinmetz MA, Pekar $\mathrm{JJ}$, et al. Transient neural activity in human parietal cortex during spatial attention shifts. Nat Neurosci. 2002;5:995-1002.

86. Shahin AJ, Picton TW, Miller LM. Brain oscillations during semantic evaluation of speech. Brain Cogn. 2009;70:259-66.

87. Spitzer B, Blankenburg F. Supramodal parametric working memory processing in humans. J Neurosci. 2012;32:3287-95.

88. Spitzer B, Gloel M, Schmidt TT, Blankenburg F. Working memory coding of analog stimulus properties in the human prefrontal cortex. Cereb Cortex. 2014:24:2229-36

89. Kawasaki M, Kitajo K, Yamaguchi Y. Dynamic links between theta executive functions and alpha storage buffers in auditory and visual working memory. Eur J Neurosci. 2010;31:1683-9.

90. Dimitriadis SI, Sun Y, Kwok K, Laskaris NA, Thakor N, Bezerianos A. Cognitive workload assessment based on the tensorial treatment of EEG estimates of cross-frequency phase interactions. Ann Biomed Eng. 2015:43:977-89.

91. Axmacher N, Henseler MM, Jensen O, Weinreich I, Elger CE, Fell J. Crossfrequency coupling supports multi-item working memory in the human hippocampus. Proc Natl Acad Sci. 2010;107:3228-33.

92. Canolty RT, Knight RT. The functional role of cross-frequency coupling. Trends Cogn Sci. 2010;14:506-15.

\section{Submit your next manuscript to BioMed Central and we will help you at every step:}

- We accept pre-submission inquiries

- Our selector tool helps you to find the most relevant journal

- We provide round the clock customer support

- Convenient online submission

- Thorough peer review

- Inclusion in PubMed and all major indexing services

- Maximum visibility for your research

Submit your manuscript at www.biomedcentral.com/submit 